\title{
THE REFINABILITY OF STEP FUNCTIONS
}

\author{
MATTHEW J. HIRN
}

(Communicated by David R. Larson)

\begin{abstract}
Refinable functions have been widely investigated because of their importance in wavelet theory and multiresolution analysis, as well as because of intrinsic interest. Problems involving refinability can be challenging and interesting problems in mathematics. Several papers have investigated refinability of splines and other classes of functions. The purpose of this paper is to develop necessary and sufficient conditions for the refinability of the class of step functions on the real line taking complex values.
\end{abstract}

\section{INTRODUCTION}

The concept of refinability of functions on the real line and in several variables in higher dimensions has been widely studied because of its importance to wavelet theory and multiresolution analysis, and because of its intrinsic interest. In particular, scaling functions for an MRA are refinable. Much work has been done by many researchers on refinability of continuous and other functions, notably splines (piecewise polynomial continuous functions). For instance, we cite the references $[2,3,4]$. In this article we consider the class of complex-valued piecewise constant functions on the real line with integer discontinuities (the step functions with integer steps). We develop necessary and sufficient conditions for the refinability of functions in this class.

We recall the definition of a (finitely) 2-refinable function on the real line.

Definition 1.1. A function $f: \mathbb{R} \rightarrow \mathbb{C}$ is 2-refinable if

$$
f(x)=\sum_{l=N_{1}}^{N_{2}} c_{l} f(2 x-l)
$$

where $c_{l} \in \mathbb{C} \forall l \in\left\{N_{1}, \ldots, N_{2}\right\}$.

After an appropriate translation a 2-refinable function $f(x)$ has the following form:

$$
f(x)=\sum_{l=0}^{N} c_{l} f(2 x-l) .
$$

We will call the set $C=\left\{c_{0}, \ldots, c_{N}\right\}$ the refinement coefficients of $f$.

Received by the editors July 20, 2004 and, in revised form, August 20, 2006.

2000 Mathematics Subject Classification. Primary 39B22.

Key words and phrases. Refinable equations, step function.

(C) 2007 American Mathematical Society Reverts to public domain 28 years from publication 
In particular we will be interested in the refinability of step functions $f: \mathbb{R} \rightarrow \mathbb{C}$,

$$
f(x)=\sum_{j=1}^{M} d_{j} \chi_{\left[a_{j}, b_{j}\right)}(x)
$$

where $M \in\{1,2,3, \ldots\}, d_{j} \in \mathbb{C}, d_{j} \neq 0 \quad \forall j \in\{1, \ldots, M\}$, and $a_{j}, b_{j} \in \mathbb{R}, a_{j}<$ $b_{j} \leq a_{j+1} \forall j \in\{1, \ldots, M\}$.

In the proof of Theorem 2.1 we will see that all refinable functions of the type in equation (2) in fact have the form:

$$
f(x)=\sum_{l=1}^{N} k_{l} \chi_{[l-1, l)}(x)
$$

where $N \in\{1,2,3, \ldots\}$ and $k_{l} \in \mathbb{C} \forall l \in\{1, \ldots, N\}$.

Let $K=\left\{k_{1}, \ldots, k_{N}\right\}$ denote the nonzero values of $f$.

Without loss of generality we can assume $k_{1} \neq 0$ and $k_{N} \neq 0$. The proof of Theorem 2.1 also shows that the value of $N$ must be the same in (1) and (3). For clarity of exposition we assume this from the start. For simplicity, we will assume $k_{1}=1$, since any multiple of a solution to (1) is also a solution.

A simple example of a refinable step function is $f(x)=\chi_{[0,1)}(x)-\chi_{[1,2)}(x)$ where $f(x)=f(2 x)+2 f(2 x-1)+f(2 x-2)$.

We will be looking at these functions from an algebraic standpoint as well as their Fourier transforms. In particular, we will examine the relationships between three sets of data that determine these functions: the values that the function takes $(K)$, the function's refinability constants $(C)$, and the function's Fourier transform. Using these relations we will come up with a complete classification of these functions.

It should be noted that the result of section 2 is contained in a more general result given in [4]. However, the result given here is obtained in a simpler manner. Furthermore, the results of section 3 are new, as are the tables given in section 4 .

I would like to thank Professor Robert Strichartz for all the help and guidance he has given me concerning this paper. Furthermore, I would also like to thank Professor David Larson for his help with this research, as well as Texas A\&M University and the NSF for funding me during the 2003 Research Experience for Undergraduates. Finally, I'd like to thank Professor Yang Wang for his useful email correspondence concerning the early stages of this project.

\section{Classifying Piecewise constant functions that are Refinable}

The most straightforward way to start classifying refinable step functions is to look at the set of $2 N$ algebraic equations that must necessarily be satisfied. Assuming that $f$ satisfies equations (1) and (3), this set of equations is

for $1 \leq n \leq N$,

$$
k_{\lceil n / 2\rceil}=\sum_{l=1}^{n} c_{l-1} k_{n-l+1}
$$

and for $N<n \leq 2 N$

$$
k_{\lceil n / 2\rceil}=\sum_{l=1}^{2 N-n+1} c_{N-l+1} k_{n-N+l-1} .
$$


Using a normalization $k_{1}=1$ one has $2 N$ equations and $2 N$ variables $\left(k_{2}, \ldots, k_{N}\right.$ and $\left.c_{0}, \ldots, c_{N}\right)$. Using equation (4) with $n=1$ and equation (5) with $n=2 N$, one can see that $c_{0}=1$ and $c_{N}=1$. Furthermore, using just the first $N$ equations, we can see that the $c$ 's determine the $k$ 's when assuming $k_{1}=1$ :

$$
k_{n}=k_{\lceil n / 2\rceil}-\left(c_{1} k_{n-1}+\ldots+c_{n-1} k_{1}\right), \quad 2 \leq n \leq N .
$$

Also, the $k$ 's determine the $c^{\prime}$ 's, $c_{0}=c_{N}=1$ :

$$
c_{n}=k_{\lceil(n+1) / 2\rceil}-\left(k_{n+1}+c_{1} k_{n}+\ldots+c_{n-1} k_{2}\right), \quad 1 \leq n \leq N-1 .
$$

We will now define the Fourier transform, $\mathcal{F}$, dilation, $D^{j}$, and translation, $T^{j}$, of a function $f$ as follows:

$$
\begin{aligned}
& \mathcal{F}[f](\lambda)=\hat{f}(\lambda)=\frac{1}{\sqrt{2 \pi}} \int_{-\infty}^{\infty} f(t) e^{-i \lambda t} d t, \\
& D^{j}[f](x)=\frac{f\left(2^{j} x\right)}{2^{j / 2}} \\
& T^{j}[f](x)=f(x-j) .
\end{aligned}
$$

Theorem 2.1. Let $p \in \mathbb{C}[z]$, where $\mathbb{C}[z]$ is defined as the set of all complex polynomials. Also, we restrict $z=e^{-i \lambda}, \lambda \in \mathbb{R}$. Then $\frac{p(z)}{i \lambda \sqrt{2 \pi}}$ is the Fourier transform of a refinable step function if and only if $\exists q \in \mathbb{C}[z]$ such that $p\left(z^{2}\right)=p(z) q(z)$ and 1 is a root of $p(z)$.

Proof. Assume that $f$ satisfies equations (1) and (2). Equation (1) implies

$$
\begin{aligned}
f(x / 2) & =\sum_{l=0}^{N} c_{l} f(x-l), \\
\frac{1}{\sqrt{2}} D^{-1}[f](x) & =\sum_{l=0}^{N} c_{l} T^{l}[f](x) .
\end{aligned}
$$

Note that $\hat{D}=D^{-1}$ and $\hat{T}^{l}=M_{e^{-i l \lambda}}$ (multiply by $e^{-i l \lambda}$ ). Therefore, taking the Fourier transform of both sides gives for the left hand side

$$
\mathcal{F}\left[\frac{1}{\sqrt{2}} D^{-1}[f](t)\right](\lambda)=\mathcal{F}[f(t / 2)](\lambda)=2 \hat{f}(2 \lambda)
$$

and for the right hand side

$$
\mathcal{F}\left[\sum_{l=0}^{N} c_{l} T^{l}[f](t)\right](\lambda)=\left(\sum_{l=0}^{N} c_{l} e^{-i l \lambda}\right) \hat{f}(\lambda)=m(\lambda) \hat{f}(\lambda),
$$

where $m(\lambda)=q(z)=\sum_{l=0}^{N} c_{l} z^{l}, z=e^{-i \lambda}$, and thus $m(\lambda) \in \mathbb{C}[z]$. Equating both sides gives

$$
2 \hat{f}(2 \lambda)=\hat{f}(\lambda) m(\lambda)
$$

Equation (2) implies

$$
\begin{aligned}
\hat{f}(\lambda) & =\frac{1}{\sqrt{2 \pi}} \sum_{j=1}^{M} d_{j} \int_{a_{j}}^{b_{j}} e^{-i \lambda t} d t \\
& =\frac{-1}{i \lambda \sqrt{2 \pi}}\left(\sum_{j=1}^{N} d_{j}\left(z^{b_{j}}-z^{a_{j}}\right)\right) .
\end{aligned}
$$


By equation (6) we have

$$
\frac{\sum_{j=1}^{N} d_{j}\left(z^{2 b_{j}}-z^{2 a_{j}}\right)}{\sum_{j=1}^{N} d_{j}\left(z^{b_{j}}-z^{a_{j}}\right)}=\sum_{l=0}^{N} c_{l} z^{l} .
$$

Carrying out the division in the left hand side of equation (7) gives

$$
z^{b_{M}}+z^{a_{M}}+\sum_{j=1}^{M-1} \frac{d_{M-j}}{d_{M}}\left(z^{a_{M-j}}-z^{b_{M-j}}\right)+[\text { other order terms in } z]=\sum_{l=0}^{N} c_{l} z^{l} .
$$

Since $d_{j} \neq 0 \quad \forall j=\{1, \ldots, M\}$ we see $a_{j}, b_{j} \in \mathbb{Z} \quad \forall j=\{1, \ldots, M\}$. Thus all refinable step functions must satisfy equation (3). We now complete the proof using this fact. Equation (3) implies

$$
\begin{aligned}
\hat{f}(\lambda) & =\frac{1}{\sqrt{2 \pi}} \sum_{l=1}^{N} k_{l} \int_{l-1}^{l} e^{-i \lambda t} d t \\
& =\frac{-1}{i \lambda \sqrt{2 \pi}}\left(\sum_{l=1}^{N} k_{l}\left(z^{l}-z^{l-1}\right)\right) \\
& =\frac{-p(z)}{i \lambda \sqrt{2 \pi}}
\end{aligned}
$$

where

$$
p(z)=\sum_{l=1}^{N} k_{l}\left(z^{l}-z^{l-1}\right)=(z-1) \sum_{l=1}^{N} k_{l} z^{l-1} .
$$

Thus $p(z)$ has 1 as a zero and from equation (6) we know

$$
p\left(z^{2}\right)=p(z) q(z) \text {. }
$$

The converse is obtained by simply taking the inverse Fourier transform of $\frac{p(z)}{i \lambda \sqrt{2 \pi}}$, which will give one a piecewise constant function. Thus the Fourier transform of this function satisfies equation (6) and it is refinable.

An important thing to note from the proof of this theorem is that the coefficients of $\frac{p(z)}{z-1}$ are the values that function takes, while the coefficients of $q(z)$ are the refinement constants.

Corollary 2.2. Let $p \in \mathbb{C}[z]$. Then $p(z)$ satisfies the conditions of Theorem 2.1 if and only if a.) for every root, $\alpha$, of $p(z)$ with multiplicity $m$, then $\alpha^{2}$ is also a root with multiplicity $\geq m$; and b.) 1 is a root of $p(z)$.

Proof. Since $p(z)$ is of degree $N$, write $p(z)$ in terms of its distinct zeroes $\left\{\alpha_{1}, \ldots, \alpha_{n}\right\}, n \leq N$, each with multiplicity $m_{l}$ :

$$
p(z)=k_{N} \prod_{l=1}^{n}\left(z-\alpha_{l}\right)^{m_{l}} .
$$

If $p(z)$ satisfies Theorem 2.1, then equation (9) holds and we know that

$$
\frac{p\left(z^{2}\right)}{p(z)}=\frac{k_{N} \prod\left(z^{2}-\alpha_{l}\right)^{m_{l}}}{k_{N} \prod\left(z-\alpha_{l}\right)^{m_{l}}}=q(z) \in \mathbb{C}[z] .
$$


Therefore each multiplicative term in the denominator must cancel with a term in the numerator and we have one direction. For the converse, we see $p(z)$ must satisfy equation (9) under such conditions and thus satisfies Theorem 2.1.

Corollary 2.3. Let $p \in \mathbb{C}[z]$. If $p(z)$ satisfies the conditions of Theorem 2.1 , then each root $\alpha$ of $p(z)$ has the form $\alpha=e^{2 \pi i \frac{r}{s}}$ where $r, s \in \mathbb{Z}$.

Proof. By Corollary 2.2, if $\alpha$ is a root, then $\alpha^{2}$ is a root. Since $p(z)$ has finitely many roots, we see $|\alpha|=1$ and thus $\alpha=e^{i \lambda}$ for some $\lambda \in \mathbb{R}$. Using the same ideas, we are left with two cases:

I. $\alpha^{2^{a}}=e^{2^{a} i \lambda}=1$ for some $a \in \mathbb{Z}, a \geq 0$. Thus there exists $d \in \mathbb{Z}$ such that $2^{a} i \lambda=2 \pi i d$, which gives $i \lambda=2 \pi i \frac{b}{2^{a}}$.

II. $\alpha^{2^{a}}=\alpha^{2^{a+b}}$ for some $a, b \in \mathbb{Z}, a \geq 0, b>0$. Thus there exists $d \in \mathbb{Z}$ such that $2^{a} i \lambda=2^{a+b} i \lambda+2 \pi i d$, which gives $i \lambda=2 \pi i \frac{d}{2^{a}-2^{a+b}}$.

In summary, a refinable step function is completely determined by any one of the following three:

i) The function value at each step, $K=\left\{k_{1}, \ldots, k_{N}\right\}$.

ii) The refinement coefficients, $C=\left\{c_{0}, \ldots, c_{N}\right\}$.

iii) The roots of $p(z), A=\left\{\alpha_{1}, \ldots, \alpha_{n}\right\}$, along with their multiplicities.

Given one of these sets, we can compute the other two sets:

$K \rightarrow p(z)$, which gives $A$ by $(8) \rightarrow \frac{p\left(z^{2}\right)}{p(z)}$, which gives $C$ by $(9)$;

$C \rightarrow$ equations (4) and (5), which gives $K$;

$A \rightarrow k_{N} \frac{1}{z-1} \prod\left(z-\alpha_{l}\right)^{m_{l}}$ where $-1=k_{N} \prod\left(-\alpha_{l}\right)^{m_{l}}$, which then gives $K$ by $(8)$.

Corollaries 2.2 and 2.3 characterize the possible sets $A$.

\section{Miscellaneous properties of Refinable Step funCtions}

Theorem 3.1. For any refinable step function, the refinement coefficients satisfy the following property:

$$
c_{l}=\overline{c_{N-l}} \quad \forall l \in\{0, \ldots,\lfloor N / 2\rfloor\} .
$$

Proof. Let $\Gamma=\left\{\gamma_{1}, \ldots, \gamma_{N}\right\}$ be the zeroes (not necessarily distinct) of $\frac{p\left(z^{2}\right)}{p(z)}$. Thus we have

$$
\frac{p\left(z^{2}\right)}{p(z)}=\prod_{l=1}^{N}\left(z-\gamma_{l}\right)=\sum_{l=0}^{N} \sigma_{l}(\Gamma) z^{l}=\sum_{l=0}^{N} c_{l} z^{l},
$$

where $\sigma_{l}(\Gamma)$ are the elementary symmetric functions.

$$
\begin{aligned}
& \sigma_{0}(\Gamma)=\gamma_{1} \gamma_{2} \cdots \gamma_{N}, \\
& \sigma_{1}(\Gamma)=\sum_{l=1}^{N} \gamma_{1} \gamma_{2} \cdots \gamma_{l-1} \gamma_{l+1} \cdots \gamma_{N}, \text { where } \gamma_{0}=\gamma_{N+1}=1, \\
& \vdots \\
& \sigma_{N-1}(\Gamma)=\sum_{l=1}^{N} \gamma_{l}, \\
& \sigma_{N}(\Gamma)=1 .
\end{aligned}
$$

Since $c_{l}=\sigma_{l}(\Gamma)$ and $c_{0}=1$ we have $\gamma_{1} \gamma_{2} \cdots \gamma_{N}=1$. Furthermore, since $\left|\gamma_{l}\right|=1, \gamma_{l}^{-1}=\overline{\gamma_{l}}$. Combining the last two statements gives $\sigma_{l}(\Gamma)=\overline{\sigma_{N-l}(\Gamma)}$ and thus $c_{l}=\overline{c_{N-l}}$.

We can also distinguish which polynomials give rise to real valued functions. 
Theorem 3.2. Let $f$ be a refinable step function with Fourier transform $\frac{p(z)}{i \lambda \sqrt{2 \pi}}$. Then $f$ is real valued if and only if for every zero $e^{i \lambda}$ of $p(z)$ with multiplicity $m$, $e^{-i \lambda}$ is a zero as well with the same multiplicity.

Proof. Assume for $p(z)$ that for every zero $e^{i \lambda}$ with multiplicity $m, e^{-i \lambda}$ is a zero as well with the same multiplicity. Then for each such pair

$$
\left(z-e^{i \lambda}\right)^{m}\left(z-e^{-i \lambda}\right)^{m}=\left(z^{2}-\left(e^{i \lambda}+e^{-i \lambda}\right) z+1\right)^{m}=\left(z^{2}-2 \cos \lambda z+1\right)^{m} .
$$

Thus the coefficients of $f$ are real, so $f$ is real valued.

Now assume $f$ is real valued. Thus $\left\{k_{1}, \ldots, k_{N}\right\}$ are all real numbers. Assume that $e^{i \lambda}$ is a zero of $p(z)$, for $e^{i \lambda} \neq \pm 1$. Since $\left(z-e^{i \lambda}\right)$ divides $p(z)=$ $(z-1) \sum_{l=1}^{N} k_{l} z^{l-1}$, this gives $\sum_{l=1}^{N} k_{l} e^{(l-1) i \lambda}=0$. Since the $k_{l}$ 's are real numbers, when we take the complex conjugate we obtain $p\left(e^{-i \lambda}\right)=0$. Similarly, by considering derivatives of $p$, we can show that the multiplicities are equal.

We can further classify the real valued functions as either even or odd, and can determine when for each. Let a complex number be called an even root of a polynomial if it is a root with even multiplicity or if it is not a root at all; call the number an odd root of the polynomial if it is a root with odd multiplicity.

Theorem 3.3. Let $f$ be a real valued refinable step function. Then $f$ must be either an even or odd function about $N / 2$. In particular, we have two cases:

I) If $N$ is even, then:

-1 is an odd root $\Leftrightarrow f$ is an even function about $N / 2$,

-1 is an even root $\Leftrightarrow f$ is an odd function about $N / 2$.

II) If $N$ is odd, then:

-1 is an odd root $\Leftrightarrow f$ is an odd function about $N / 2$,

-1 is an even root $\Leftrightarrow f$ is an even function about $N / 2$.

Proof. First we see that $k_{N}= \pm 1$ (assuming $k_{1}=1$ ) by writing $p(z)$ in terms of its roots. (For this proof each $\alpha_{l}$ is not necessarily distinct.)

$$
\begin{aligned}
p(z)=k_{N} \prod_{l=1}^{N}\left(z-\alpha_{l}\right) & =k_{N}\left(z^{N}+\ldots+(-1)^{N} \alpha_{1} \cdots \alpha_{N}\right) \\
& =k_{N}(z-1)\left(z^{N-1}+\ldots+(-1)^{N-1} \alpha_{1} \cdots \alpha_{N}\right) .
\end{aligned}
$$

By Theorem 3.2 we know that $\alpha_{1} \cdots \alpha_{N}= \pm 1$. Therefore,

$$
k_{1}=(-1)^{N-1} k_{N} \alpha_{1} \cdots \alpha_{N}= \pm k_{N}=1 .
$$

In particular, if $N$ is even and if -1 is an even root, then $k_{N}=-1$, while if -1 is an odd root, then $k_{N}=1$. Alternatively, if $N$ is odd and if -1 is an even root, then $k_{N}=1$, while if -1 is an odd root, then $k_{N}=-1$. The converses easily follow by using the same logic backwards. We now complete the proof that $f$ must be an even or odd function about $N / 2$.

From equations (4) and (5) with $n=1$ and $n=2 N-1$ we have

$$
\begin{gathered}
c_{1} k_{1}+c_{0} k_{2}=k_{1} \quad \text { and } \quad c_{N-1} k_{N}+c_{N} k_{N-1}=k_{N}, \\
c_{1}=\frac{k_{1}-k_{2}}{k_{1}} \quad \text { and } \quad c_{N-1}=\frac{k_{N}-k_{N-1}}{k_{N}} .
\end{gathered}
$$


By Theorem 3.1 we have $c_{1}=c_{N-1}$ and thus

$$
\begin{aligned}
\frac{k_{1}-k_{2}}{k_{1}} & =\frac{k_{N}-k_{N-1}}{k_{N}}, \\
k_{1} k_{N-1} & =k_{2} k_{N}, \\
\frac{k_{1}}{k_{N}} & =\frac{k_{2}}{k_{N-1}} .
\end{aligned}
$$

Therefore $\frac{k_{2}}{k_{N-1}}= \pm 1$. Using both ratios and equations (4) and (5) with $n=2$ and $n=2 N-2$ we get $\frac{k_{3}}{k_{N-2}}=\frac{k_{1}}{k_{N}}= \pm 1$. Continue this process to get that all the ratios are equal, and thus $f$ must either be even or odd.

\section{Sets of Refinable Step functions}

Tables 1-4 were computed using the system of algebraic equations detailed in section 2 and solving them in MATLAB.

TABle 1. Corresponding sets of $K, C$, and $A$ of refinable step functions for $N=3$

\begin{tabular}{|l|l|l|}
\hline$k_{1}, k_{2}, k_{3}$ & $c_{0}, c_{1}, c_{2}, c_{3}$ & values of $\beta$ for which $p\left(e^{2 \pi i \beta}\right)=0$ \\
\hline $1,0,-1$ & $1,1,1,1$ & $0,0, \frac{1}{2}$ \\
\hline $1,1,1$ & $1,0,0,1$ & $0, \frac{1}{3}, \frac{2}{3}$ \\
\hline $1,-2,1$ & $1,3,3,1$ & $0,0,0$ \\
\hline $1,1+i, i$ & $1,-i, i, 1$ & $0, \frac{1}{4}, \frac{1}{2}$ \\
\hline $1,1-i,-i$ & $1, i,-i, 1$ & $0, \frac{1}{2}, \frac{3}{4}$ \\
\hline
\end{tabular}

TABle 2. Corresponding sets of $K, C$, and $A$ of refinable step functions for $N=5$ (real valued functions only)

\begin{tabular}{|l|l|l|}
\hline$k_{1}, k_{2}, k_{3}, k_{4}, k_{5}$ & $c_{0}, c_{1}, c_{2}, c_{3}, c_{4}, c_{5}$ & values of $\beta$ for which $p\left(e^{2 \pi i \beta}\right)=0$ \\
\hline $1,0,1,0,1$ & $1,1,-1,-1,1,1$ & $0, \frac{1}{6}, \frac{1}{3}, \frac{2}{3}, \frac{5}{6}$ \\
\hline $1,0,-2,0,1$ & $1,1,2,2,1,1$ & $0,0,0, \frac{1}{2}, \frac{1}{2}$ \\
\hline $1,1,1,1,1$ & $1,0,0,0,0,1$ & $0, \frac{1}{5}, \frac{2}{5}, \frac{3}{5}, \frac{4}{5}$ \\
\hline $1,-4,6,-4,1$ & $1,5,10,10,5,1$ & $0,0,0,0,0$ \\
\hline $1,-1,0,-1,1$ & $1,2,1,1,2,1$ & $0,0,0, \frac{1}{3}, \frac{2}{3}$ \\
\hline $1,2,3,2,1$ & $1,-1,1,1,-1,1$ & $0, \frac{1}{3}, \frac{1}{3}, \frac{2}{3}, \frac{2}{3}$ \\
\hline $1,0,0,0,-1$ & $1,1,0,0,1,1$ & $0,0, \frac{1}{4}, \frac{1}{2}, \frac{3}{4}$ \\
\hline $1,1,0,-1,-1$ & $1,0,1,1,0,1$ & $0,0, \frac{1}{3}, \frac{1}{2}, \frac{2}{3}$ \\
\hline $1,-2,0,2,-1$ & $1,3,4,4,3,1$ & $0,0,0,0, \frac{1}{2}$ \\
\hline
\end{tabular}


TABle 3. Corresponding sets of $K, C$, and $A$ of refinable step functions for $N=4$

\begin{tabular}{|l|l|l|}
\hline$k_{1}, k_{2}, k_{3}, k_{4}$ & $c_{0}, c_{1}, c_{2}, c_{3}, c_{4}$ & values of $\beta$ for which $p\left(e^{2 \pi i \beta}\right)=0$ \\
\hline $1,1,-1,-1$ & $1,0,2,0,1$ & $0,0, \frac{1}{2}, \frac{1}{2}$ \\
\hline $1,0,0,-1$ & $1,1,0,1,1$ & $0,0, \frac{1}{3}, \frac{2}{3}$ \\
\hline $1,-3,3,-1$ & $1,4,6,4,1$ & $0,0,0,0$ \\
\hline $1,-1,-1,1$ & $1,2,2,2,1$ & $0,0,0, \frac{1}{2}$ \\
\hline $1,2,2,1$ & $1,-1,2,-1,1$ & $0, \frac{1}{3}, \frac{1}{2}, \frac{2}{3}$ \\
\hline $1,1,1,1$ & $1,0,0,0,1$ & $0, \frac{1}{4}, \frac{1}{2}, \frac{3}{4}$ \\
\hline $1, i,-1,-i$ & $1,1-i, 0,1+i, 1$ & $0,0, \frac{1}{4}, \frac{1}{2}$ \\
\hline $1,-i,-1, i$ & $1,1+i, 0,1-i, 1$ & $0,0, \frac{1}{2}, \frac{3}{4}$ \\
\hline $1, \frac{1}{2}+i \frac{\sqrt{7}}{2},-\frac{1}{2}+i \frac{\sqrt{7}}{2},-1$ & $1, \frac{1}{2}-i \frac{\sqrt{7}}{2},-1, \frac{1}{2}+i \frac{\sqrt{7}}{2}, 1$ & $0, \frac{1}{7}, \frac{2}{7}, \frac{4}{7}$ \\
\hline $1, \frac{1}{2}-i \frac{\sqrt{7}}{2},-\frac{1}{2}-i \frac{\sqrt{7}}{2},-1$ & $1, \frac{1}{2}+i \frac{\sqrt{7}}{2},-1, \frac{1}{2}-i \frac{\sqrt{7}}{2}, 1$ & $0, \frac{3}{7}, \frac{5}{7}, \frac{6}{7}$ \\
\hline $1, \frac{1}{2}-i \frac{\sqrt{3}}{2}, \frac{1}{2}-i \frac{\sqrt{3}}{2},-\frac{1}{2}-i \frac{\sqrt{3}}{2}$ & $1, \frac{1}{2}+i \frac{\sqrt{3}}{2},-1, \frac{1}{2}-i \frac{\sqrt{3}}{2}, 1$ & $0, \frac{1}{3}, \frac{2}{3}, \frac{5}{6}$ \\
\hline $1, \frac{1}{2}+i \frac{\sqrt{3}}{2}, \frac{1}{2}+i \frac{\sqrt{3}}{2},-\frac{1}{2}+i \frac{\sqrt{3}}{2}$ & $1, \frac{1}{2}-i \frac{\sqrt{3}}{2},-1, \frac{1}{2}+i \frac{\sqrt{3}}{2}, 1$ & $0, \frac{1}{6}, \frac{1}{3}, \frac{2}{3}$ \\
\hline $1, \frac{2+\sqrt{2}}{2}+i \frac{2-\sqrt{2}}{2}, \sqrt{2}+i, \frac{\sqrt{2}}{2}+i \frac{\sqrt{2}}{2}$ & $1,-\frac{\sqrt{2}}{2}-i \frac{2-\sqrt{2}}{2}, \sqrt{2},-\frac{\sqrt{2}}{2}+i \frac{2-\sqrt{2}}{2}, 1$ & $0, \frac{1}{4}, \frac{1}{2}, \frac{5}{8}$ \\
\hline $1, \frac{2-\sqrt{2}}{2}+i \frac{2+\sqrt{2}}{2},-\sqrt{2}+i,-\frac{\sqrt{2}}{2}-i \frac{\sqrt{2}}{2}$ & $1, \frac{\sqrt{2}}{2}-i \frac{2+\sqrt{2}}{2},-\sqrt{2}, \frac{\sqrt{2}}{2}+i \frac{2+\sqrt{2}}{2}, 1$ & $0, \frac{1}{8}, \frac{1}{4}, \frac{1}{2}$ \\
\hline $1, \frac{2+\sqrt{2}}{2}-i \frac{2-\sqrt{2}}{2}, \sqrt{2}-i, \frac{\sqrt{2}}{2}-i \frac{\sqrt{2}}{2}$ & $1,-\frac{\sqrt{2}}{2}+i \frac{2-\sqrt{2}}{2}, \sqrt{2},-\frac{\sqrt{2}}{2}-i \frac{2-\sqrt{2}}{2}, 1$ & $0, \frac{3}{8}, \frac{1}{2}, \frac{3}{4}$ \\
\hline $1, \frac{2-\sqrt{2}}{2}-i \frac{2+\sqrt{2}}{2},-\sqrt{2}-i,-\frac{\sqrt{2}}{2}+i \frac{\sqrt{2}}{2}$ & $1, \frac{\sqrt{2}}{2}+i \frac{2+\sqrt{2}}{2},-\sqrt{2}, \frac{\sqrt{2}}{2}-i \frac{2+\sqrt{2}}{2}, 1$ & $0, \frac{1}{2}, \frac{3}{4}, \frac{7}{8}$ \\
\hline
\end{tabular}


TABle 4. Corresponding sets of $K, C$, and $A$ of refinable step functions for $N=6$ (real valued functions only)

\begin{tabular}{|l|l|l|}
\hline$k_{1}, k_{2}, k_{3}, k_{4}, k_{5}, k_{6}$ & $c_{0}, c_{1}, c_{2}, c_{3}, c_{4}, c_{5}, c_{6}$ & values of $\beta$ for which $p\left(e^{2 \pi i \beta}\right)=0$ \\
\hline $1,0,-1,-1,0,1$ & $1,1,1,2,1,1,1$ & $0,0,0, \frac{1}{3}, \frac{1}{2}, \frac{2}{3}$ \\
\hline $1,-1,0,0,-1,1$ & $1,2,1,0,1,2,1$ & $0,0,0, \frac{1}{4}, \frac{1}{2}, \frac{3}{4}$ \\
\hline $1,3,5,5,3,1$ & $1,-2,4,-4,4,-2,1$ & $0, \frac{1}{3}, \frac{1}{3}, \frac{1}{2}, \frac{2}{3}, \frac{2}{3}$ \\
\hline $1,-3,2,2,-3,1$ & $1,4,7,8,7,4,1$ & $0,0,0,0,0, \frac{1}{2}$ \\
\hline $1,1-\sqrt{2}, 2-\sqrt{2}, 2-\sqrt{2}, 1-\sqrt{2}, 1$ & $1, \sqrt{2}, 1-\sqrt{2},-2,1-\sqrt{2}, \sqrt{2}, 1$ & $0, \frac{1}{8}, \frac{1}{4}, \frac{1}{2}, \frac{3}{4}, \frac{7}{8}$ \\
\hline $1,1+\sqrt{2}, 2+\sqrt{2}, 2+\sqrt{2}, 1+\sqrt{2}, 1$ & $1,-\sqrt{2}, 1+\sqrt{2},-2,1+\sqrt{2},-\sqrt{2}, 1$ & $0, \frac{1}{4}, \frac{3}{8}, \frac{1}{2}, \frac{5}{8}, \frac{3}{4}$ \\
\hline $1,1,1,1,1,1$ & $1,0,0,0,0,0,1$ & $0, \frac{1}{6}, \frac{1}{3}, \frac{1}{2}, \frac{2}{3}, \frac{5}{6}$ \\
\hline $1,1,-2,-2,1,1$ & $1,0,3,0,3,0,1$ & $0,0,0, \frac{1}{2}, \frac{1}{2}, \frac{1}{2}$ \\
\hline $1,2,3,3,2,1$ & $1,-1,1,0,1,-1,1$ & $0, \frac{1}{4}, \frac{1}{3}, \frac{1}{2}, \frac{2}{3}, \frac{3}{4}$ \\
\hline $1,2,2,2,2,1$ & $1,-1,2,-2,2,-1,1$ & $0, \frac{1}{5}, \frac{2}{5}, \frac{1}{2}, \frac{3}{5}, \frac{4}{5}$ \\
\hline $1,1,1,-1,-1,-1$ & $1,0,0,2,0,0,1$ & $0,0, \frac{1}{3}, \frac{1}{3}, \frac{2}{3}, \frac{2}{3}$ \\
\hline $1,-1,1,-1,1,-1$ & $1,2,0,-2,0,2,1$ & $0,0, \frac{1}{6}, \frac{1}{3}, \frac{2}{3}, \frac{5}{6}$ \\
\hline $1,-2,1,-1,2,-1$ & $1,3,3,2,3,3,1$ & $0,0,0,0, \frac{1}{3}, \frac{2}{3}$ \\
\hline $1,2,1,-1,-2,-1$ & $1,-1,3,-2,3,-1,1$ & $0,0, \frac{1}{3}, \frac{1}{2}, \frac{1}{2}, \frac{2}{3}$ \\
\hline $1,1,0,0,-1,-1$ & $1,0,1,0,1,0,1$ & $0,0, \frac{1}{4}, \frac{1}{2}, \frac{1}{2}, \frac{3}{4}$ \\
\hline $1,0,0,0,0,-1$ & $1,1,0,0,0,1,1$ & $0,0, \frac{1}{5}, \frac{2}{5}, \frac{3}{5}, \frac{4}{5}$ \\
\hline $1,-5,10,-10,5,-1$ & $1,6,15,20,15,6,1$ & $0,0,0,0,0,0$ \\
\hline $1,-1,-2,2,1,-1$ & $1,2,3,4,3,2,1$ & $0,0,0,0, \frac{1}{2}, \frac{1}{2}$ \\
\hline
\end{tabular}




\section{REFERENCES}

[1] A.S. Cavaretta, W. Dahman and C.A. Micchelli, Stationary subdivision, Memoirs Amer. Math. Soc. \# 453, 93 (1991) 1-182. MR1079033 (92h:65017)

[2] Xin-Rong Dai, De-Jun Fend, and Yang Wang, Classification of Refinable Splines, Applied and Computational Harmonic Analysis.

[3] I. Daubechies, Ten Lectures on Wavelets, CBMS 61 (Society of Industrial and Applied Mathematics, Philadelphia, PA, 1992). MR1162107 (93e:42045)

[4] Wayne Lawton, S.L. Lee and Zuowei Shen, Characterization of compactly supported refinable splines, Advances in Computational Mathematics 3(1995)137-145. MR1314906 (95m:41020)

Department of Mathematics, Cornell University, Ithaca, New York

Current address: Department of Mathematics, University of Maryland, College Park, Maryland

E-mail address: hirn@math.umd.edu 